\title{
Detección de Aedes albopictus (Skuse) (Diptera: Culicidae) en el municipio de Istmina, Chocó, Colombia
}

\author{
José Joaquín Carvajal ${ }^{1,2,3}$, Nildimar Alves Honorio ${ }^{2,3}$, Silvia Patricia Díaz ${ }^{4}$, Edinso Rafael Ruiz², \\ Jimmy Asprilla ${ }^{4}$, Susanne Ardila ${ }^{5}$, Gabriel Parra-Henao \\ 1 Laboratório de Doenças Parasitarias, Instituto Oswaldo Cruz, Fundação Oswaldo Cruz, Rio de Janeiro, Brasil \\ 2 Laboratório de Transmissores de Hematozoários, Instituto Oswaldo Cruz, Fundação Oswaldo Cruz, Rio de \\ Janeiro, Brasil \\ 3 Núcleo Operacional Sentinela de Mosquitos Vetores-NOSMOVE/FIOCRUZ, Rio de Janeiro, Brasil \\ 4 Secretaría de Salud del Chocó, Quibdó, Colombia \\ 5 Laboratorio de Entomología, Instituto Nacional de Salud, Bogotá, D.C., Colombia \\ 6 Centro de Investigación en Salud para el Trópico, Facultad de Medicina, Universidad Cooperativa de Colombia, \\ Santa Marta, Colombia
}

Introducción. Aedes albopictus se encuentra ampliamente distribuido en el mundo. Su introducción en las Américas ocurrió en 1985 y se le considera vector potencial de los virus del dengue y uno de los principales vectores del virus del chikungunya. En Colombia, su primer reporte fue en Leticia, Amazonas, en 1998, seguido de Buenaventura, Valle del Cauca, en 2001, Barrancabermeja, Santander, en 2010 y Medellín, Antioquia, en 2011. La especie se ha reportado en diez departamentos del país.

Objetivo. Notificar el hallazgo de A. albopictus en el municipio de Istmina, Chocó, y dar cuenta de su importancia en salud pública.

Materiales y métodos. En enero de 2015 se inspeccionaron criaderos de los barrios de San Agustín, Santa Genoveva y Subestación del municipio de Istmina para la detección de formas inmaduras de Aedes spp. Las larvas recolectadas fueron identificadas en la Unidad de Entomología del Laboratorio de Salud Pública Departamental de Chocó y confirmadas en el Laboratorio de Entomología de la Red Nacional de Laboratorios del Instituto Nacional de Salud en Bogotá.

Resultados. Se encontraron doce larvas de $A$. albopictus en criaderos ubicados en los barrios Subestación y San Agustín en el municipio de Istmina.

Conclusión. La detección de $A$. albopictus en el municipio de Istmina resalta la importancia del fortalecimiento de las estrategias de vigilancia entomológica continua a nivel municipal y departamental en el país, y especialmente en Istmina y los municipios aledaños.

Palabras clave: Aedes, dengue, virus chikungunya, vigilancia, Colombia.

doi: http://dx.doi.org/10.7705/biomedica.v36i3.2805

\section{Detection of Aedes albopictus (Skuse) (Diptera: Culicidae) in the municipality of Istmina, Chocó, Colombia}

Introduction: Aedes albopictus is widely distributed around the world. Its introduction to the Americas occurred in 1985 and it is considered a potential vector of dengue viruses and one of the principal vectors of chikungunya virus. In Colombia, this species was reported for the first time in Leticia (Amazonas) in 1998, followed by Buenaventura (Valle del Cauca) in 2001, Barrancabermeja (Santander) in 2010, and Medellín (Antioquia) in 2011. So far, this species has been reported in ten departments of the country. Objective: To report the finding of $A$. albopictus in the city of Istmina, Chocó, and its implications for public health.

Materials and methods: In January 2015, we conducted an inspection of immature stages of Aedes spp. in breeding sites in the neighborhoods of San Agustín, Santa Genoveva and Subestación in Istmina, Chocó. The immature stages collected in this municipality were identified at the Unidad de Entomología of the Laboratorio de Salud Pública Departamental de Chocó, and confirmed by the Laboratorio de Entomología, Red Nacional de Laboratorios, Instituto Nacional de Salud, in Bogotá.

\footnotetext{
Contribución de los autores:

Nildimar Alves Honório, José Joaquín Carvajal y Edinso Mosquera: desarrollo de los muestreos de campo, procesamiento de material, identificación taxonómica y escritura del manuscrito

Jimmy Asprilla: desarrollo de los muestreos de campo y procesamiento de material

Susanne Ardila: confirmación taxonómica de las especies

Gabriel Parra-Henao: desarrollo de los muestreos de campo, procesamiento de material, confirmación taxonómica de las especies y escritura del manuscrito
} 
Results: In January 2015, twelve $A$. albopictus larvae were found in the breeding sites located in Subestación and San Agustín neighborhoods.

Conclusions: The occurrence of $A$. albopictus in the municipality of Istmina underlines the importance of strengthening continuous entomological surveillance strategies at national and local levels in the country, especially in Istmina and its surrounding municipalities.

Key words: Aedes, dengue, chikungunya virus, surveillance, Colombia. doi: http://dx.doi.org/10.7705/biomedica.v36i3.2805

Las arbovirosis transmitidas por Aedes aegypti y A. albopictus (Diptera: Culicidae), como el dengue y el chikungunya, se consideran un importante problema de salud pública en Colombia y en el mundo $(1,2)$.

Aedes albopictus, también conocido como "mosquito tigre asiático", es una especie oriunda del sureste asiático, donde actúa como vector primario de los virus del dengue en diversos países $(2,3)$. Se ha demostrado que $A$. albopictus es vector de otras arbovirosis como el chikungunya (4-6), la fiebre del Nilo occidental $(7,8)$, la encefalitis equina del este (3) y la encefalitis japonesa (9).

Sin embargo, en Latinoamérica esta especie aún no se ha incriminado como vector primario del dengue (2), a pesar de que el hallazgo de formas inmaduras del mosquito infectadas con DENV-1 sugiere la transmisión transovárica en Brasil (10) y Buenaventura, Colombia (11). Asímismo, en México se registró la infección natural de machos adultos con DENV-2 (12).

Aedes albopictus es una especie ampliamente distribuida en las regiones tropicales y en los países templados, y está presente en 29 países de África, Europa, Asia y América (13). La colonización de diversos países del mundo por $A$. albopictus está relacionada con el transporte pasivo de sus huevos a través del intenso comercio de llantas y plantas acuáticas ornamentales (14). Su introducción y propagación en el continente americano durante la década de los ochenta, se presentó por la acelerada expansión del tráfico aéreo y marítimo, la deficiente vigilancia entomológica, las condiciones ambientales favorables para su reproducción y su adaptabilidad a los mismos depósitos que sirven como criaderos de $A$. aegypti en ambientes domésticos y peridomésticos $(15,16)$.

\footnotetext{
Correspondencia:

Gabriel Parra-Henao, Carretera Troncal del Caribe, Centro de Investigación en Salud para el Trópico, Facultad de Medicina, Universidad Cooperativa de Colombia, Sector Mamatoco, Santa Marta, Colombia

Teléfono: (575) 420 9604, extensión 5538

gparrahenao@gmail.com
}

Recibido: 01/06/15; aceptado: 26/03/16
En las Américas, la presencia del vector fue informada por primera vez en Hawaii en 1903 (17); posteriormente, en agosto de 1985, en Houston, Texas, se detectaron formas inmaduras en llantas usadas provenientes de Asia (18). En Suramérica se detectó por primera vez en el municipio de Itaguaí, Rio de Janeiro, en 1986, introducido en tocones de bambú importados del Japón destinados a construir "quebravientos" para fines agrícolas (19). En Colombia, la presencia de ocho ejemplares adultos de $A$. albopictus se registró por primera vez en 1998 en el municipio de Leticia, Amazonas (20).

Posteriormente, se reportó en Buenaventura en 2001 (21), en Cali en 2007 (22), en Barrancabermeja en 2010 (Gutiérrez M, Almeida O, Barrios H, Herrera J, Ramírez M, Rondón L, et al. Hallazgo de Aedes albopictus (Diptera: Culicidae) en el municipio de Barrancabermeja, Colombia. Biomédica. 2011;31:26. Memorias, XX Congreso Latinoamericano de Parasitología, Bogotá, D.C., mayo de 2011), en Medellín (23) y en Condoto, Chocó (Confirmación Taxonómica, Muestra No. 2011-32284, Laboratorio de Entomología, RNLINS), en 2011. Hasta la fecha, el Laboratorio de Entomología del Instituto Nacional de Salud ha registrado su presencia en 51 localidades de 10 departamentos del país (figura 1).

Aedes albopictus tiene una amplia tolerancia ecológica y frecuentemente se encuentra en ambientes silvestres con vegetación natural y baja densidad de población humana, aunque también puede encontrarse en ambientes suburbanos con una relativa cobertura vegetal y preferentemente en el peridomicilio (24-27). En algunos estudios de caracterización de los criaderos de $A$. albopictus en el municipio de Leticia, Colombia, los autores observaron que la especie se presentó en el 59,1 $\%$ de los criaderos inspeccionados, con preferencia por las llantas y los depósitos inservibles, y que era tolerante a ambientes acuáticos con poco oxígeno disuelto $(5,6 \%)$, y valores elevados de conductividad $(291,3 \mathrm{~ms} / \mathrm{cm})$ y de turbidez (461 FTU), lo cual demuestra su rápida adaptación a las condiciones de los criaderos disponibles que favorecen el establecimiento de la especie (28). 


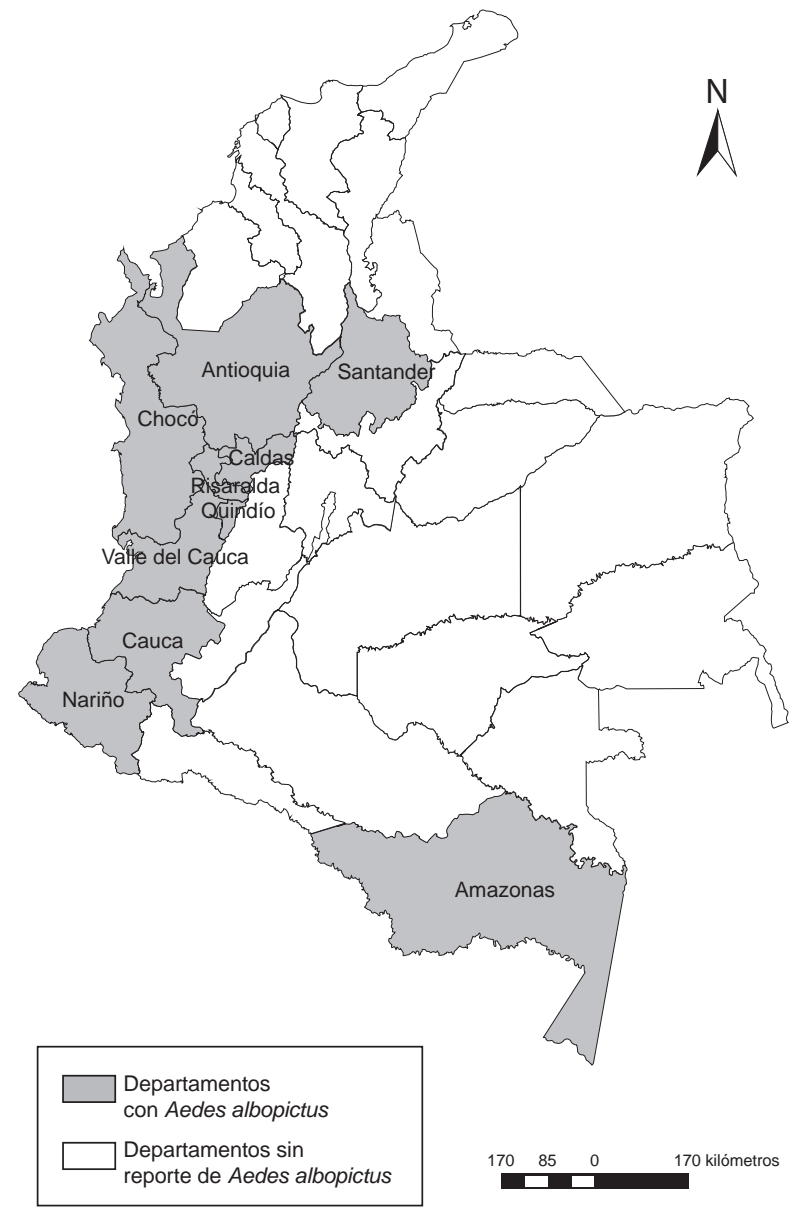

Figura 1. Presencia de Aedes albopictus en Colombia Fuente: Instituto Geográfico Agustín Codazzi, escala 1:100.000

Esta especie tiene hábitos exofágicos y exofílicos, actividad diurna y bimodal, discordancia gonotrófica y oviposición en depósitos naturales y artificiales $(29,30)$. Asimismo, es bastante heterogénea en lo que respecta a su alimentacion, pues puede alimentarse de sangre de otros vertebrados además del humano (31-34) y, especialmente, por la capacidad de poner sus huevos sin necesidad de alimentación sanguínea previa (35-37). Por otro lado, en diversos estudios se ha demostrado que los huevos de $A$. albopictus pueden entrar en diapausa, la cual se activa por estímulos específicos que reducen la morfogénesis, mediados principalmente por el fotoperíodo y la temperatura $(32,38)$.

La rápida expansión de $A$. albopictus, asociada con la gran competencia vectorial de las poblaciones de esta especie para los virus del dengue y del chikungunya, confirman la necesidad de implementar estrategias de vigilancia entomológica continuas (39-41). El objetivo del presente estudio fue notificar el hallazgo de $A$. albopictus en el municipio de Istmina, Chocó, y dar cuenta de su importancia en salud pública.

\section{Materiales y métodos}

El departamento del Chocó está localizado en la región del Pacífico colombiano, y limita con los océanos Atlántico y Pacífico. Tiene una extensión de $46.530 \mathrm{~km}^{2}$, que corresponden al $4 \%$ de la extensión geográfica del país. Aproximadamente, el $90 \%$ del territorio está conformado por zonas especiales de conservación, entre las cuales sobresalen el Tapón del Darién y las cuencas de los ríos Atrato y San Juan. Según la clasificación climática de Holdridge, presenta un clima intertropical lluvioso con más de $9.000 \mathrm{~mm}$ de precipitación anual acumulada y una temperatura promedio de $27^{\circ} \mathrm{C}$. Los centros urbanos más importantes son Quibdó (capital), Istmina, Condoto, Acandí y Bahía Solano, los cuales albergan, en su conjunto, alrededor del $50 \%$ de la población del departamento (42). El $87 \%$ del territorio de Istmina está ubicado sobre la cuenca media del río San Juan y, el $13 \%$ restante, sobre la del río Atrato, y en él predominan la extracción forestal y minera. El área urbana está compuesta principalmente por viviendas de tipo residencial de tres habitaciones y dos pisos, por lo general, con paredes y pisos de madera y tejas de asbesto o cemento (43).

La presencia de $A$. albopictus en este municipio (figura 2) se registró durante el desarrollo de las actividades de campo del proyecto denominado "Descripción etno-epidemiológica y entomológica del dengue en población afrodescendiente en el municipio de Istmina, departamento de Chocó, Colombia", realizado por investigadores de la Universidad Cooperativa de Colombia (Santa Marta) y del Instituto Oswaldo Cruz (Rio de Janeiro), en cooperación con el programa de enfermedades transmitidas por vectores de la Unidad de Entomología de la Secretaría de Salud Departamental del Chocó y la Secretaría de Salud de Istmina.

Como parte de dichas actividades, entre el 16 y el 19 de enero de 2015, se hizo la búsqueda activa de criaderos de Aedes en los barrios Subestación, San Agustín y Santa Genoveva, con el fin de caracterizarlos. En cada vivienda seleccionada se informaba a un adulto responsable sobre las actividades por desarrollar y, una vez que aprobaba y firmaba el consentimiento, se pedía su autorización para ingresar a la vivienda e inspeccionar todos los depósitos detectados en 


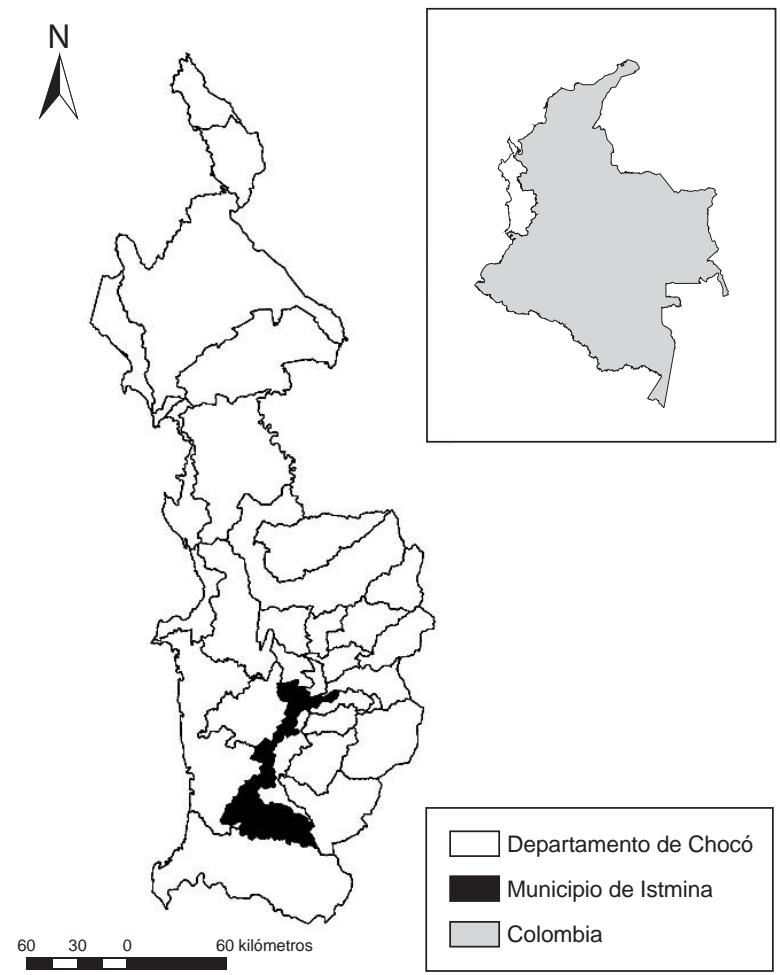

Figura 2. Municipio de Istmina, Chocó, Colombia Fuente: Instituto Geográfico Agustín Codazzi, escala 1:100.000

el domicilio y el peridomicilio en busca de formas inmaduras. En los depósitos en los que estas se encontraban se tomaban muestras representativas para su posterior identificación en el laboratorio. Todo el material recolectado fue identificado en el Laboratorio Departamental de Salud Pública del Chocó y su confirmación taxonómica se hizo en el Laboratorio de Entomología de la Subdirección de la Red Nacional de Laboratorios del Instituto Nacional de Salud.

\section{Resultados}

El primer hallazgo de $A$. albopictus en el Chocó se reportó en el municipio de Condoto en diciembre del 2011, en un depósito ubicado en el domicilio; se trataba de tres larvas recolectadas durante el levantamiento del índice aédico por parte de los agentes del programa de Enfermedades Transmitidas por Vectores de la Secretaría de Salud Departamental del Chocó en diciembre de ese año y confirmado taxonómicamente por el Grupo de Entomología de la Red Nacional de Laboratorios del Instituto Nacional de Salud (código de muestra No. 2011-32284). Sin embargo, la especie no se registró nuevamente en el departamento (datos sin publicar).
Entre los días 16 y 19 de enero de 2015 se inspeccionaron 72 viviendas y se encontraron seis criaderos con formas inmaduras de Aedes spp. Una vez identificados los especímenes en el laboratorio, se encontró que en cuatro de los criaderos había larvas de $A$. aegypti, en uno, larvas de $A$. albopictus y en el otro, larvas de las dos especies (cuadro 1).

Todos los recipientes positivos se hallaban en el peridomicilio de viviendas de los barrios de Subestación y San Agustín, ubicados en el área periurbana del municipio. Se destacó la presencia de larvas de $A$. albopictus en un barril utilizado para almacenamiento de agua de lluvia en el patio de una vivienda en el barrio Subestación y en un recipiente metálico descartado hallado en la parte trasera de una vivienda en el barrio San Agustín, colindante con el área de reserva forestal del municipio (figura 3).

\section{Discusión}

Aunque se desconozcan los medios que propiciaron la colonización de $A$. albopictus en el Chocó, es evidente la importancia del transporte pasivo del mosquito en la dispersión de la especie, pues los municipios de Condoto e Istmina son puntos importantes de entrada y salida de mercancías por vía aérea, terrestre y fluvial, con una gran movilidad humana y un mayor intercambio económico y comercial, especialmente entre Buenaventura e Istmina, por el río San Juan $(14,44)$. Cabe resaltar que el panorama epidemiológico del Chocó se ve agravado por su proximidad con otros departamentos infestados por el mismo vector (figura 1) y por el aumento de casos notificados de chikungunya (1.004 casos) en otros municipios del departamento durante el 2015 (45).

Los depósitos encontrados en el peridomicilio de las viviendas eran principalmente recipientes desechados 0 utilizados para el almacenamiento de agua, lo cual puede indicar deficiencias en los servicios básicos de suministro de agua y recolección de residuos sólidos; esta situación, además del conocimiento limitado sobre la ecología de los mosquitos y la gran vulnerabilidad social y cultural, favorece el mantenimiento o el establecimiento de Aedes en estos municipios (46-48).

La dinámica de las infecciones tropicales como el dengue y el chikungunya, y de sus vectores, así como su expansión geográfica horizontal y vertical, están fuertemente influenciadas por la temperatura, la precipitación, la humedad relativa y los efectos del cambio climático global (49-52). La 
Cuadro 1. Depósitos con larvas de Aedes spp. en el municipio de Istmina, Chocó

\begin{tabular}{lccccc}
\hline \multirow{2}{*}{ Fecha } & Barrio & Tipo de depósito & $\begin{array}{c}\text { Larvas de } \\
\boldsymbol{A} \text {. albopictus }\end{array}$ & $\begin{array}{c}\text { Larvas de } \\
\boldsymbol{A} \text {. aegypti }\end{array}$ & Localización \\
\hline $16 / 01 / 2015$ & Subestación & Diverso (inservible) & 0 & 19 & $\begin{array}{c}\text { Peridomicilio } \\
17 / 01 / 2015\end{array}$ \\
$17 / 01 / 2015$ & Subestación & Tanque bajo & 2 & 0 & Peridomicilio \\
$17 / 01 / 2015$ & Subestación & Tanque bajo & 0 & 6 & Peridomicilio \\
$17 / 01 / 2015$ & Subestación & Tanque bajo & 0 & 2 & Peridomicilio \\
$19 / 01 / 2015$ & Subestación & Tanque bajo & 0 & 10 & Peridomicilio \\
\hline
\end{tabular}

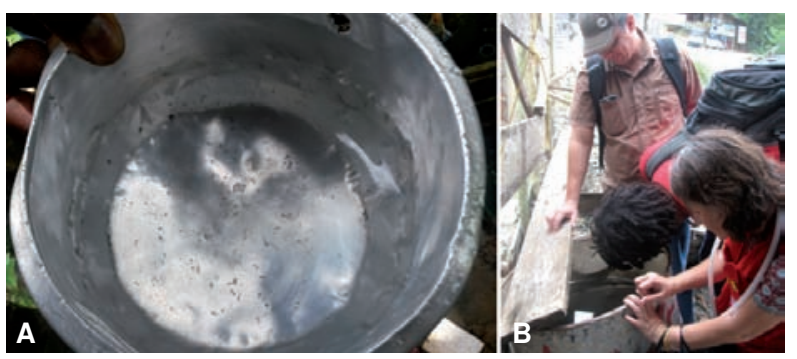

Figura 3. Criaderos positivos con formas inmaduras de Aedes albopictus en el municipio de Istmina. A. Recipiente metálico localizado en el peridomicilio (barrio San Agustín). B. Barril localizado en el peridomicilio (barrio Subestación)

precipitación, por ejemplo, contribuye al incremento de las poblaciones de estos mosquitos al aumentar la tasa de eclosión, la diversidad y la disponibilidad de recipientes naturales y artificiales, y reducir la toxicidad por la renovación del agua $(53,54)$. La temperatura afecta el comportamiento de picada, la mortalidad de adultos, y las tasas de desarrollo larval y de replicación del virus $(32,38,55,56)$. En el Chocó, las altas temperaturas y precipitaciones durante todo el año favorecen la dispersión y pueden acelerar el establecimiento de las poblaciones de esta especie (57), lo cual resalta la importancia de una vigilancia entomológica adecuada y continua, y la implementación e intensificación de estrategias de vigilancia centinela en los municipios colindantes, donde es posible que ya haya presencia de la especie $(58,59)$.

En las Americas, $A$. albopictus se ha encontrado coexistiendo con $A$. aegypti en áreas suburbanas con abundancias similares, lo cual también se ha reportado en la mayoría de los municipios de Colombia $(25,26,60,61)$. Por otra parte, en algunos estudios se ha demostrado la existencia de un aparente 'desplazamiento' de A. aegypti en ambientes urbanos donde $A$. albopictus gradualmente ha conseguido expulsar a su competidor $(62,63)$. Otros estudios reportan que la coexistencia de las dos especies puede estar modulada por las fluctuaciones ambientales y los mecanismos de estabilización que las contrarrestan y, en algunos casos, pueden coexistir hasta en el $40 \%$ de los criaderos (64-67). Sin embargo, se requieren estudios locales para evaluar los efectos de las poblaciones de $A$. albopictus y $A$. aegypti en la dinámica de transmisión de estas enfermedades que, al parecer, dependen del contexto.

Aedes albopictus puede ser más competente en la transmisión del virus del chikungunya, como es el caso de la variante del virus E1-226A, preferencialmente seleccionada por este vector para su replicación $(5,6,41)$. En estudios Ilevados a cabo en diez países en América por Vega-Rúa, et al. (39), se demostró que los tres genotipos del virus del chikungunya (genotipos ECSA, asiático y del oeste africano) infectaban todas las poblaciones estudiadas de $A$. albopictus y podían transmitirse a través de ellas; en algunos casos alcanzaron hasta el $90 \%$ de eficiencia frente a las poblaciones de $A$. aegypti, las cuales alcanzaron solo $70 \%$ de eficiencia.

En varios estudios se sugiere la necesidad de la vigilancia de $A$. albopictus por su reconocido papel de transmisor de varias arbovirosis, como el dengue, la fiebre del Nilo occidental y el chikungunya, así como por su posible involucramiento en el mantenimiento de estos virus en la naturaleza, su modo oportunista de alimentación, su capacidad de transmisión transovárica, su mayor adaptabilidad y flexibilidad ecológica, y sobre todo, su capacidad excepcional de autogénesis $(27,29,33,36,67-70)$. En el caso de los virus del dengue, $A$. albopictus es un vector menos competente y aún no se ha incriminado en su transmisión en Latinoamérica $(3,71-75)$. Sin embargo, la infección natural con DENV-1 y DENV-2 se ha comprobado en Buenaventura, Colombia, (11) y con DENV-2 y DENV-3, en México (12). Por otra parte, también se encontraron larvas infectadas por DENV-1 en Minas Gerais, Brasil (10), y bajo condiciones de laboratorio se ha demostrado su capacidad de 
infectar y transmitir DENV-1 y DENV-2 (31,7679). Cabe resaltar que, aunque se ha demostrado que es menos competente para la transmisión de los virus del dengue en algunas regiones, $A$. albopictus podría adaptarse en el futuro y aumentar su competencia vectorial (80).

Otra particularidad importante es su potencial capacidad de actuar como puente entre los ciclos de transmisión urbano y selvático de la fiebre amarilla selvática, por encontrarse frecuentemente en áreas periurbanas $(2,30,81)$, lo cual favorecería la aparición de la fiebre del Nilo occidental en el Chocó biogeográfico, dado que es uno de los corredores de aves migratorias más importantes en el neotrópico, y se ha reportado a $A$. albopictus alimentándose de una amplia variedad de aves pertenecientes a los grupos de passeriformes, galliformes, columbiformes y ciconiformes, los cuales incluyen especies migratorias $(36,82)$.

Dado que actualmente no se cuenta con una vacuna tetravalente, aunque hay algunas en prueba, como la de Sanofi-Pasteur, cuya eficacia se está evaluando (fase 3) (83), el control vectorial continúa siendo el único método eficiente para prevenir la transmisión de los virus del dengue. En este sentido, se hace necesario continuar con la aplicación de estrategias de control vectorial sistemático, tanto para $A$. albopictus como para $A$. aegypti, y buscar alternativas de vigilancia que sean más sensibles y específicas, como las ovitrampas, y que permitan estimar efectivamente la infestación con estos dos vectores en el país $(84,85)$. Sin embargo, el éxito de las iniciativas y esfuerzos como los proyectos desarrollados por el Instituo Nacional de Salud para la vigilancia de adultos de los dos vectores de dengue y chikungunya, y de la implementación y seguimiento de los protocolos e instructivos de vigilancia entomológica, depende de la articulación de las estrategias de educación y comunicación en la comunidad, y de la capacitación continua para elevar la calidad del personal involucrado en la vigilancia sistémica de estos vectores.

En este trabajo se registró la presencia de $A$. albopictus en el Chocó, ampliándose así su distribución en el país, lo cual resalta su importancia en salud pública por ser vector de los virus del dengue, del chikungunya y de otras arbovirosis en el país.

\section{Agradecimientos}

A Silvia Mosquera, coordinadora del programa de Enfermedades Transmitidas por Vectores de la Secretaría de Salud Departamental del Chocó, y encargada de la vigilancia entomológica de dengue y chikungunya en el departamento, así como a su equipo de auxiliares, por su colaboración en las actividades de campo del proyecto. A la Secretaría de Salud Municipal de Istmina, por su colaboración en la logística durante el desarrollo del proyecto.

\section{Conflicto de intereses}

Los autores manifestamos que no existe ningún conflicto de intereses en torno a los resultados presentados.

\section{Financiación}

Este trabajo fue financiado por el Instituto Oswaldo Cruz, la Coordenação de Aperfeiçoamento de Pessoal de Nível Superior (CAPES), Fundação Carlos Filho de Amparo à Pesquisa do Estado do Rio de Janeiro, Brasil; recibió subvención de FAPERJ (N E-26/102.241/2013).

\section{Referencias}

1. Weaver SC. Arrival of chikungunya virus in the new world: Prospects for spread and impact on public health. PLoS Negl Trop Dis. 2014;8:6-9. http://dx.doi.org/10.1371/journal. pntd.0002921

2. Rúa-Uribe G, Suárez-Acosta C, Rojo RA. Implicaciones epidemiológicas de Aedes albopictus (Skuse) en Colombia. Rev Nac Salud Pública. 2012;3:328-37.

3. Gratz NG. Critical review of the vector status of Aedes albopictus. Med Vet Entomol. 2004;18:215-27. http://dx.doi. org/10.1111/j.0269-283X.2004.00513.x

4. Mangiafico JA. Chikungunya virus infection and transmission in five species of mosquito. Am J Trop Med Hyg. $1971 ; 20: 642-5$

5. Tsetsarkin KA, Vanlandingham DL, McGee CE, Higgs S. A single mutation in Chikungunya virus affects vector specificity and epidemic potential. PLoS Pathog. 2007;3:1895-906. http://dx.doi.org/10.1371/journal.ppat.0030201

6. Vazeille M, Moutailler S, Coudrier D, Rousseaux C, Khun $\mathbf{H}$, Huerre $\mathbf{M}$, et al. Two chikungunya isolates from the outbreak of La Reunion (Indian Ocean) exhibit different patterns of infection in the mosquito Aedes albopictus. PLoS One. 2007;2:e1168. http://dx.doi.org/10.1371/journal. pone.0001168

7. Turell MJ, Sardelis MR, Dohm DJ, O'guinn ML. Potential North American vectors of West Nile Virus. Ann N Y Acad Sci. 2001;951:317-24. http://dx.doi.org/10.1111/j.1749-6632. 2001.tb02707.x

8. Holick J, Kyle A, Ferraro W, Delaney RR, Iwaseczko M. Discovery of Aedes albopictus infected with West Nile virus in southeastern Pennsylvania. J Am Mosq Control Assoc. 2002;18:131.

9. Rosen L, Tesh RB, Lien JC, Cross JH. Transovarial transmission of Japanese encephalitis virus by mosquitoes. Science. 1978;199:909-11. http://dx.doi.org/10.1126/science. 203035 
10. Serufo JC, de Oca HM, Tavares VA, Souza AM, Rosa RV, Jamal MC, et al. Isolation of dengue virus type 1 from larvae of Aedes albopictus in Campos Altos city, State of Minas Gerais, Brazil. Mem do Inst Oswaldo Cruz. 1993;88:503-4. http://dx.doi.org/10.1590/S0074-02761993000300025

11. Méndez F, Barreto M, Arias JF, Rengifo G, Muñoz J, Burbano ME, et al. Human and mosquito infections by dengue viruses during and after epidemics in a dengueendemic region of Colombia. Am J Trop Med Hyg. 2006;74: 678-83.

12. Ibáñez-Bernal S, Briseño B, Mutebi JP, Argot E, Rodríguez G, Martínez-Campos C, et al. First record in America of Aedes albopictus naturally infected with dengue virus during the 1995 outbreak at Reynosa, México. Med Vet Entomol. 1997;11:305-9. http://dx.doi.org/10.1111/j.13652915.1997.tb00413.x

13. Benedict MQ, Levine RS, Hawley WA, Lounibos LP. Spread of the tiger: Global risk of invasion by the mosquito Aedes albopictus. Vector Borne Zoonotic Dis. 2007;7:76-85. http://dx.doi.org/10.1089/vbz.2006.0562.

14. Hawley WA, Reiter P, Copeland RS, Pumpuni CB, Craig GB. Aedes albopictus in North America: Probable introduction in used tires from northern Asia. Science. 1987;236:1114-6. http://dx.doi.org/10.1126/science.3576225

15. Organización Panamericana de la Salud. Aedes albopictus en las Américas. Reseñas. Bol Sanit Panam. 1987;102: 624-33.

16. Ponce G, Flores AE, Badii MH, Fernández I. Bionomía de Aedes albopictus (Skuse). Rev Salud Pública Nutr. 2004;5:1-14

17. Bonnet DD, Worcester DJ. The dispersal of Aedes albopictus in the territory of Hawaii. Am J Trop Med Hyg. 1946;26:465-76.

18. Sprenger D, Wuithiranyagool T. The discovery and distribution of Aedes albopictus in Harris County, Texas. J Am Mosq Control Assoc. 1986;2:217-9.

19. Forattini OP. Identificação de Aedes (Stegomyia) albopictus (Skuse) no Brasil. Rev Saúde Pública. 1986;20:244-5. http:// dx.doi.org/10.1590/S0034-89101986000300009

20. Vélez ID, Quiñones ML, Suárez M, Olano V, Murcia LM, Correa E, et al. Presencia de Aedes albopictus en Leticia, Amazonas, Colombia. Biomédica. 1998;18:192-8. http://dx. doi.org/10.7705/biomedica.v18i3.990

21. Suárez M. Aedes albopictus (Skuse) (Diptera: Culicidae) en Buenaventura, Colombia. Inf Quinc Epidemiológico Nac. $2001 ; 6: 221-4$.

22. Cuéllar-Jiménez ME, Velásquez-Escobar OL, GonzálezObando R. Detección de Aedes albopictus (Skuse) (Diptera: Culicidae) en la ciudad de Cali, Valle del Cauca, Colombia. Biomédica. 2007;27:273-9. http://dx.doi.org/10.7705/ biomedica.v27i2.224

23. Rúa-Uribe GL, Suárez-Acosta $C$, Londoño $V$, Sánchez J, Rojo R, Bello-Novoa B. Primera evidencia de Aedes albopictus (Skuse) (Diptera: Culicidae) en la ciudad de Medellín, Antioquia-Colombia. Revista Salud Pública de Medellín. 2011;5:89-98.

24. Honório N, Castro M. The spatial distribution of Aedes aegypti and Aedes albopictus in a transition zone, Rio de Janeiro, Brazil. Cad Saúde Pública. 2009;25:1203-14. http:// dx.doi.org/10.1590/S0102-311X2009000600003
25. Braks MA, Honório NA, Lourençqo-De-Oliveira R, Juliano SA, Lounibos LP. Convergent habitat segregation of Aedes aegypti and Aedes albopictus (Diptera: Culicidae) in southeastern Brazil and Florida. J Med Entomol. 2003;40: 785-94. http://dx.doi.org/10.1603/0022-2585-40.6.785

26. Lima-Camara TN De, Honório NA, Lourenço-de-Oliveira R. Freqüência e distribuição espacial de Aedes aegypti e Aedes albopictus (Diptera: Culicidae) no Rio de Janeiro, Brasil. Cad Saúde Pública. 2006;22:2079-84. http://dx.doi. org/10.1590/S0102-311X2006001000013

27. Marquetti MDC, Valdés V, Aguilera L. Tipificación de hábitats de Aedes albopictus en Cuba y su asociación con otras especies de culícidos, 1995-1998. Rev Cubana Med Trop. 2000;52:170-4.

28. Carvajal JJ, Moncada LI, Rodríguez MH, Pérez LDP, Olano VA. Caracterización preliminar de los sitios de cría de Aedes (Stegomyia) albopictus (Skuse, 1894) (Diptera: Culicidae) en el municipio de Leticia, Amazonas, Colombia. Biomédica. 2009;29:413-23. http://dx.doi.org/10.7705/ biomedica.v29i3.13

29. Corbet PS, Chadee DD. An improved method for detecting substrate preferences shown by mosquitoes that exhibit "skip oviposition." Physiol Entomol. 1993;18:114-8. http:// dx.doi.org/10.1111/j.1365-3032.1993.tb00457.x

30. Consoli RAGB, Lourenço-de-Oliveira R. Principais mosquitos de importância sanitária no Brasil. Rio de Janeiro: Editora FIOCRUZ; 1994. p. 228.

31. Almeida AP, Baptista SS, Sousa CA, Novo MT, Ramos HC, Panella NA, et al. Bioecology and vectorial capacity of Aedes albopictus (Diptera: Culicidae) in Macao, China, in relation to dengue virus transmission. J Med Entomol. 2005;42:41928. http://dx.doi.org/10.1093/jmedent/42.3.419

32. Delatte H, Gimonneau G, Triboire A, Fontenille D. Influence of temperature on immature development, survival, longevity, fecundity, and gonotrophic cycles of Aedes albopictus, vector of chikungunya and dengue in the Indian Ocean. J Med Entomol. 2009;46:33-41. http://dx.doi. org/10.1603/033.046.0105

33. De Lima-Camara TN. Activity patterns of Aedes aegypti and Aedes albopictus (Diptera: Culicidae) under natural and artificial conditions. Oecologia Australis. 2010;14:737-44.

34. Sullivan MF, Gould DJ, Maneechai S. Observations on the host range and feeding preferences of Aedes albopictus (Skuse). J Med Entomol. 1971;8:713-6. http://dx.doi.org/10. 1093/jmedent/8.6.713

35. Savage HM, Niebylski ML, Smith GC, Mitchell CJ, Craig GB. Host-feeding patterns of Aedes albopictus (Diptera: Culicidae) at a temperate North American site. J Med Entomol. 1993;30:27-34. http://dx.doi.org/10.1093/ jmedent/30.1.27

36. Niebylski ML, Savage HM, Nasci RS, Craig GB. Blood hosts of Aedes albopictus in the United States. J Am Mosq Control Assoc. 1994;10:447-50.

37. Klowden MJ, Chambers GM. Reproductive and metabolic differences between Aedes aegypti and Ae. albopictus (Diptera: Culicidae). J Med Entomol. 1992;29:467-71. http:// dx.doi.org/10.1093/jmedent/29.3.467

38. Pumpuni CB, Knepler J, Craig GB. Influence of temperature and larval nutrition on the diapause inducing photoperiod of Aedes albopictus. J Am Mosq Control Assoc. 1992;8:223-7. 
39. Vega-Rúa A, Zouache $K$, Girod R, Failloux A-B, Lourenço-de-Oliveira R. High level of vector competence of Aedes aegypti and Aedes albopictus from ten American countries as a crucial factor in the spread of chikungunya virus. J Virol. 2014;88:6294-306. http://dx.doi.org/10.1128/ JVI.00370-14

40. Pancetti FG, Honório NA, Urbinatti PR, Lima-Camara TN. Twenty-eight years of Aedes albopictus in Brazil: A rationale to maintain active entomological and epidemiological surveillance. Rev Soc Bras Med Trop 2015;48:87-9. http:// dx.doi.org/10.1590/0037-8682-0155-2014

41. Arias-Goeta C, Mousson L, Rougeon F, Failloux A-B. Dissemination and transmission of the E1-226V variant of chikungunya virus in Aedes albopictus are controlled at the midgut barrier level. PLoS One. 2013;8:e57548. http:// dx.doi.org/10.1371/journal.pone.0057548

42. Instituto Geográfico Agustín Codazzi (IGAC). Diccionario Geográfico de Colombia. 2014. Fecha de consulta: 15 de diciembre de 2015. Disponible en: http://www.igac.gov.co/ digeo/app/index.html

43. Rentería I, Palacios LF. Plan de gestión integral de residuos sólidos del municipio de Istmina-Chocó. Quibdó: Universidad Tecnológica del Chocó Diego Luis Cordoba; 2004. p.134.

44. Kobayashi M, Nihei N, Kurihara T. Analysis of Northern distribution of Aedes albopictus (Diptera: Culicidae) in Japan by geographical information systems. J Med Entomol. 2002;39:4-11. http://dx.doi.org/10.1603/0022-2585-39.1.4

45 Instituto Nacional de Salud. Boletín Epidemiológico Semanal: semana epidemiológica número 52 de 2015 (27 de diciembre al 02 de enero). Bogotá: Instituto Nacional de Salud; 2015.

46. Tauil PL. Urbanization and dengue ecology. Cad Saúde Pública. 2001;17 (Suppl):99-102. http://dx.doi.org/10.1590/ S0102-311X2001000700018

47. Roberts $\mathbf{C H}$, Mongkolsapaya J, Screaton G. New opportunities for control of dengue virus. Curr Opin Infect Dis. 2013;26:567-74. http://dx.doi.org/10.1097/QCO. 0000000000000016

48. Alcaldía Municipal de Istmina. Plan de desarrollo territorial del municipio de Istmina 2012-2015: "Istmina, una empresa de todos". Istmina: Alcaldía Municipal de Istmina; 2012. p. 145 .

49. Hales S, de Wet N, Maindonald J, Woodward A. Potential effect of population and climate changes on global distribution of dengue fever: An empirical model. Lancet. 2002;360:830-4. http://dx.doi.org/10.1016/S01406736(02)09964-6

50. Morrison AC, Gray K, Getis A, Astete H, Sihuincha M, Focks D, et al. Temporal and geographic patterns of Aedes aegypti (Diptera: Culicidae) production in lquitos, Perú. J Med Entomol. 2004;41:1123-42. http://dx.doi. org/10.1603/0022-2585-41.6.1123

51. Bhatt S, Gething PW, Brady OJ, Messina JP, Farlow AW, Moyes CL, et al. The global distribution and burden of dengue. Nature. 2013;496:504-7. http://dx.doi.org/10.1038/ nature 12060

52. Fischer D, Thomas SM, Suk JE, Sudre B, Hess A, Tjaden NB, et al. Climate change effects on Chikungunya transmission in Europe: Geospatial analysis of vector's climatic suitability and virus' temperature requirements. Int J Health Geogr. 2013;12:51. http://dx.doi.org/10.1186/1476072X-12-51

53. Sunahara T, Mogi M. Priority effects of bamboo-stump mosquito larvae: Influences of water exchange and leaf litter input. Ecol Entomol. 2002;27:346-54. http://dx.doi. org/10.1046/j.1365-2311.2002.00417.x

54. Koenraadt CJM, Harrington LC. Flushing effect of rain on container-inhabiting mosquitoes Aedes aegypti and Culex pipiens (Diptera: Culicidae). J Med Entomol. 2008;45:28-35. http://dx.doi.org/10.1093/jmedent/45.1.28

55. Watts DM, Burke DS, Harrison BA, Whitmire RE, Nisalak A. Effect of temperature on the vector efficiency of Aedes aegypti for dengue 2 virus. Am J Trop Med Hyg.1987;36: $143-52$.

56. Pinto E, Coelho M, Oliver L, Massad E. The influence of climate variables on dengue in Singapore. Int $\mathrm{J}$ Environ Health Res. $2011 ; 21: 415-26$. http://dx.doi.org/10.1080/0960 3123.2011.572279.

57. Rochlin I, Ninivaggi DV, Hutchinson ML, Farajollahi A. Climate change and range expansion of the asian tiger mosquito (Aedes albopictus) in Northeastern USA: Implications for public health practitioners. PLoS ONE. 2013;8: e60874. http://dx.doi.org/10.1371/journal.pone.0060874

58. Organização Panamericana da Saúde. Enfoques ecossistêmicos em saúde: perspectivas para sua adoção no Brasil e países da América Latina. Brasília: Ministerio da Saúde; 2009. p. 44

59. Salvatella-Agrelo R. Aedes aegypti, Aedes albopictus (Diptera: Culicidae) y su papel como vectores en las Américas. La situación de Uruguay. Rev Med Uruguay. 1996;12:28-36.

60. Rey JR, Lounibos P. Ecología de Aedes aegypti y Aedes albopictus en América y transmisión de enfermedades. Biomédica. 2015;35:177-85. http://dx.doi.org/10.7705/ biomedica.v35i2.2514

61. Juliano SA. Species introduction and replacement among mosquitoes: Interspecific resource competition or apparent competition?. Ecology. 1998;79:255-68. http://dx.doi.org/10. 1890/0012-9658(1998)079[0255:SIARAM]2.0.CO;2

62. Honório NA, Lourenǫ-de-Oliveiria R. Frequency of Aedes aegypti and Aedes albopictus larvae and pupae in traps, Brazil. Rev Saúde Pública. 2001;35:385-91. http://dx.doi. org/10.1590/S0034-89102001000400009

63. Briegel H, Timmermann SE. Aedes albopictus (Diptera: Culicidae): Physiological aspects of development and reproduction. J Med Entomol. 2001;38:566-71. http://dx.doi. org/10.1603/0022-2585-38.4.566

64. Simard F, Nchoutpouen E, Toto JC, Fontenille D. Geographic distribution and breeding site preference of Aedes albopictus and Aedes aegypti (Diptera: Culicidae) in Cameroon, Central Africa. J Med Entomol. 2005;42:726-31. http://dx.doi.org/10.1093/jmedent/42.5.726

65 Honório NA, Cabello PH, Codeço CT, Lourenço-deOliveira R. Preliminary data on the performance of Aedes albopictus immatures developing in water-field tires in Rio de Janeiro. Mem Inst Oswaldo Cruz. 2006;101:225-8. http:// dx.doi.org/10.1590/S0074-02762006000200017 
66. Bagny-Beilhe L, Delatte $H$, Juliano SA, Fontenille D, Quilici S. Ecological interactions in Aedes species on Reunion Island. Med Vet Entomol. 2013;27:387-97. http:// dx.doi.org/10.1111/j.1365-2915.2012.01062.x

67. O'Neal PA, Juliano SA. Seasonal variation in competition and coexistence of Aedes mosquitoes: Stabilizing effects of egg mortality or equalizing effects of resources?. J Anim Ecol. 2013;82:256-65. http://dx.doi.org/10.1111/j.1365-2656. 2012.02017.x

68. Bat-Miriam M, Craig GB. Mutants in Aedes albopictus (Diptera: Culicidae). Mosq News.1966;26:13-22.

69. Cui KL. The autogeny of Aedes albopictus in Guangzhou arca. Acta Entomol Sin.1982; 25:256-9.

70. Moore CG, Mitchell CJ. Aedes albopictus in the United States: Ten-year presence and public health implications. Emerg Infect Dis. 1997;3:329-34. http://dx.doi.org/10.3201/ eid0303.970309

71. Liew C, Curtis CF. Horizontal and vertical dispersal of dengue vector mosquitoes, Aedes aegypti and Aedes albopictus, in Singapore. Med Vet Entomol. 2004;18:35160. http://dx.doi.org/10.1111/j.0269-283X.2004.00517.x

72. Favier C, Schmit D, Müller-Graf CDM, Cazelles B, Degallier N, Mondet B, et al. Influence of spatial heterogeneity on an emerging infectious disease: The case of dengue epidemics. Proc Biol Sci. 2005;272:1171-7. http:// dx.doi.org/10.1098/rspb.2004.3020

73. Linthicum KJ, Kramer VL, Madon MB, Fujioka K. Introduction and potential establishment of Aedes albopictus in California in 2001. J Am Mosq Control Assoc. 2003;19:301-8.

74. Degallier N, Marcus J, Teixeira S, Soares S, Regilene D, Pinto SCF, et al. Aedes albopictus may not be vector of dengue virus in human epidemics in Brazil. Rev Saúde Pública. 2003;37:386-7. http://dx.doi.org/10.1590/S003489102003000300019

75. Lambrechts L, Scott TW, Gubler DJ. Consequences of the expanding global distribution of Aedes albopictus for dengue virus transmission. PLoS Negl Trop Dis. 2010;4: e646. http://dx.doi.org/10.1371/journal.pntd.0000646

76. Effler PV, Pang L, Kitsutani P, Vorndam V, Nakata M, Ayers T, et al. Dengue fever, Hawaii, 2001-2002. Emerg Infect Dis. 2005;11:742-9. http://dx.doi.org/10.3201/eid1105. 041063
77. Calisher CH, Nuti M, Lazuick JS, Ferrari JD, Kappus KD. Dengue in the Seychelles. Bull World Health Organ. 1981;59:619-22.

78. Moore PR, Johnson PH, Smith GA, Ritchie SA, van Den Hurk AF. Infection and dissemination of dengue virus type 2 in Aedes aegypti, Aedes albopictus, and Aedes scutellaris from the Torres Strait, Australia. J Am Mosq Control Assoc. 2007;23:383-8. http://dx.doi.org/10.2987/5598.1

79. Rudnick A, Chan YC. Dengue type 2 virus in naturally infected Aedes albopictus mosquitoes in Singapore. Science. 1965;149:638-9. http://dx.doi.org/10.1126/science. 149.3684 .638

80. Moncayo AC, Fernández Z, Ortíz D, Diallo M, Sall A, Hartman S, et al. Dengue emergence and adaptation to peridomestic mosquitoes. Emerg Infect Dis. 2004;10:1790-6. http://dx.doi.org/10.3201/eid1010.030846

81. Massad E, Coutinho FA, Burattini MN, López LF. The risk of yellow fever in a dengue-infested area. Trans $\mathrm{R}$ Soc Trop Med Hyg. 2001;95:370-4.

82. Bayly NJ, Cárdenas-Ortiz L, Rubio M, Gómez C. Migration of raptors, swallows and other diurnal migratory birds through the Darien of Colombia. Ornitol Neotrop. 2014;25:63-71.

83. Capeding MR, Tran NH, Hadinegoro SR, Ismail HI, Chotpitayasunondh T, Chua MN, et al. Clinical efficacy and safety of a novel tetravalent dengue vaccine in healthy children in Asia: A phase 3, randomised, observer-masked, placebo-controlled trial. Lancet. 2014;384:1358-65. http:// dx.doi.org/10.1016/S0140-6736(14)61060-6

84. Alarcón ÉP, Segura ÁM, Rúa-Uribe G, Parra-Henao G. Evaluación de ovitrampas para vigilancia y control de Aedes aegypti en dos centros urbanos del Urabá antioqueño. Biomédica. 2014;34:409-24. http://dx.doi.org/10.7705/ biomedica.v34i3.2134

85. Instituto Oswaldo Cruz. Avaliação de armadilhas para a vigilância entomológica de Aedes aegypti com vistas à elaboração de novos índices de infestação. Nota Técnica N.․ 3/2014/IOC-FIOCRUZ/DIRETORIA. Rio de Janeiro: Fiocuz; 2014. 